\title{
Soluble CD14 in cerebrospinal fluid is associated with markers of inflammation and axonal damage in untreated HIV-infected patients: a retrospective cross-sectional study
}

Sofie Jespersen ${ }^{1}$, Karin Kæreby Pedersen ${ }^{1}$, Birgitta Anesten², Henrik Zetterberg ${ }^{3,4}$, Dietmar Fuchs ${ }^{5}$, Magnus Gisslén ${ }^{2}$, Lars Hagberg ${ }^{2}$, Marius Trøseid ${ }^{6}$ and Susanne Dam Nielsen ${ }^{1 *}$

\begin{abstract}
Background: HIV-associated cognitive impairment has declined since the introduction of combination antiretroviral treatment (CART). However, milder forms of cognitive impairment persist. Inflammation in the cerebrospinal fluid (CSF) has been associated with cognitive impairment, and CSF neurofilament light chain protein (NFL) and CSF neopterin concentrations are increased in those patients. Microbial translocation in HIV infection has been suggested to contribute to chronic inflammation, and lipopolysaccharide (LPS) and soluble CD14 (SCD14) are markers of microbial translocation and the resulting monocyte activation, respectively. We hypothesised that microbial translocation contributes to inflammation and axonal damage in the central nervous system (CNS) in untreated HIV infection.

Methods: We analyzed paired samples of plasma and CSF from 62 HIV-infected, untreated patients without cognitive symptoms from Sahlgrenska University Hospital, Gothenburg, Sweden. Measurements of neopterin and NFL in CSF were available from previous studies. Plasma and CSF SCD14 was measured using ELISA (R\&D, Minneapolis, MN), and plasma and CSF LPS was measured using LAL colorimetric assay (Lonza, Walkersville, MD, USA). Univariate and multivariate regression analyses were performed.

Results: LPS in plasma was associated with plasma SCD14 $(r=0.31, P=0.015)$, and plasma SCD14 was associated with CSF SCD14 $(r=0.32, P=0.012)$. Furthermore, CSF SCD14 was associated with NFL $(r=0.32, P=0.031)$ and neopterin $(r=0.32, P=0.012)$ in CSF. LPS was not detectable in CSF. In a multivariate regression model CSF SCD14 remained associated with NFL and neopterin after adjusting for age, CD4+ cell count, and HIV RNA in CSF.

(Continued on next page)
\end{abstract}

\footnotetext{
* Correspondence: sdn@dadlnet.dk

This data has not been presented at any meeting or conference.

'Department of Infectious Diseases, Viro-immunology Research Unit,

Rigshospitalet, University of Copenhagen, Blegdamsvej 9, DK 2100

Copenhagen $\varnothing$, Denmark

Full list of author information is available at the end of the article
} 
(Continued from previous page)

Conclusions: In a group of untreated, HIV-infected patients LPS was associated with SCD14 in plasma, and plasma sCD14 was associated CSF sCD14. CSF sCD14 were associated with markers of CNS inflammation and axonal damage. This suggest that microbial translocation might be a driver of systemic and CNS inflammation. However, LPS was not detectable in the CSF, and since SCD14 is a marker of monocyte activation SCD14 may be increased due to other causes than microbial translocation. Further studies regarding cognitive impairment and biomarkers are warranted to fully understand causality.

Keywords: HIV, CNS inflammation, Microbial translocation, sCD14, NFL, Neopterin

\section{Background}

Prior to introduction of combination antiretroviral treatment (cART) a large proportion of HIV-infected patients developed severe neurocognitive disorders $[1,2]$. With the introduction of cART, the incidence of severe HIVassociated neurocognitive disorders decreased, while milder types persists $[3,4]$.

A number of studies suggest that cognitive impairment may be caused by inflammation in the central nervous system (CNS) [5-8]. Thus, biomarkers of inflammation in the cerebrospinal fluid (CSF) have been associated with cognitive impairment. One such biomarker is neopterin, a marker of monocyte activation which has been found to be elevated in the CSF of HIV-infected patients. The concentration of neopterin decreases after initiation of cART, although it does not reach the concentrations observed in HIV-uninfected persons [9, 10], hence indicating persistent CNS inflammation [11]. Neurofilament light chain protein (NFL) is a marker of CNS axonal damage that has been associated with CNS inflammation. NFL is increased in HIV-infected patients with cognitive impairment or opportunistic CNS infections [12-17].

Systemic inflammation in HIV-infected patients has been reported repeatedly [18-20] and is believed to be partly caused by microbial translocation. Microbial translocation and immune activation are predictors of disease progression and non-AIDS-related morbidity in HIV infection [21-23]. During acute HIV infection, the gastrointestinal mucosa is depleted of $\mathrm{T}$ cells leading to translocation of microbial products such as lipopolysaccharide (LPS) into the systemic circulation [24-28]. LPS activates cells of the innate immune system by binding to Toll-like receptor-4 (TLR-4). CD14 needs to be present in order for LPS to activate the receptor. CD14 is expressed on monocytes, macrophages, and neutrophils, and upon stimulation by LPS, as well as other microbial products, CD14 is secreted and cleaved from the cells as soluble CD14 (sCD14) [29].

We hypothesised that microbial translocation contributes to inflammation in the CNS of HIV-infected patients. We therefore aimed to investigate if markers of microbial translocation (LPS) and monocyte activation (sCD14) were detectable in CSF and to determine possible associations between these markers and markers of inflammation and axonal damage in the CNS (NFL, neopterin) in untreated HIV-infected patients.

\section{Methods}

Participants

Since 1985, HIV-infected patients in Gothenburg, Sweden have been included in a longitudinal study that includes serial sampling of CSF, plasma, and serum. This cohort has previously been described in detail [14]. Lumbar punctures are performed in a standardized manner at least annually and more frequently in connection with antiretroviral treatment initiation or change in treatment. CSF and blood samples are stored at $-80{ }^{\circ} \mathrm{C}$ in batches. Both asymptomatic and symptomatic patients are included, and as of December 2014 the cohort included 494 patients who had undergone 1805 lumbar punctures. Patients were clinical assessed for neurocognitive function (reporting no challenges in regard to daily living or work).

From this cohort 20 untreated HIV-infected patients without CNS opportunistic infection but with pleocytosis the CSF $(>5$ cells $/ \mu \mathrm{L})$ indicating HIV-induced inflammation was included in a pilot study in order to determine if it was possible to detect sCD14 and LPS in CSF during HIV infection. The 20 participants from the pilot study did not participate in the following analyses.

Afterwards, 62 untreated, HIV-infected patients without cognitive symptoms or CNS opportunistic infections with samples collected between 2004 and 2014 were selected for further analyses. Untreated patients were selected to avoid confounding effects of cART on microbial translocation and inflammation. 30 patients had pleocytosis.

In order to determine if $\mathrm{CD} 4+$ cell count affected either microbial translocation or monocyte activation, patients were selected to have either CD4+ cell count $>350$ cells/ $\mu \mathrm{L}(n=31, \mathrm{CD} 4+$ cell count range 380-1400, median 530 cells $/ \mu \mathrm{L})$ or CD $4+$ cell count $<200$ cells $/ \mu \mathrm{L}(n=31, \mathrm{CD} 4+$ cell count range $0-190$, median 100 cells $/ \mu \mathrm{L})$. Groups were matched on gender, age, and CSF leucocyte count. The selection was done randomly from the list of patients fulfilling the inclusion criteria (Table 1). Five patients with 
Table 1 Characteristics of the study population

\begin{tabular}{|c|c|}
\hline \multicolumn{2}{|l|}{ Untreated HIV-infected patients } \\
\hline Characteristics & Total sample $(n=62)$ \\
\hline Age (years, range) & $39(24-68)$ \\
\hline Gender (male, $n(\%))$ & $32(52)$ \\
\hline CD4+ cell count (cells/ $\mu \mathrm{L})$ & $190(98-530)$ \\
\hline HIV RNA blood (copies/mL) & 39587 (8799-172541) \\
\hline HIV RNA CSF (copies/mL) & $4690(749-28150)$ \\
\hline 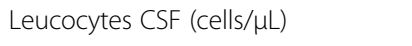 & $5(1-9)$ \\
\hline Serum albumin $(g / L)$ & $39(36-42)$ \\
\hline CSF albumin (mg/L) & $165(136-243)$ \\
\hline Albumin ratio & $3.5(4.1-5.9)$ \\
\hline \multicolumn{2}{|l|}{ Mode of transmission $(n(\%))$ : } \\
\hline Heterosexual & $49(79)$ \\
\hline MSM & $11(18)$ \\
\hline IV drug users & $2(3)$ \\
\hline \multicolumn{2}{|l|}{ AIDS defining diseases $(n(\%))$ : } \\
\hline Pneumocystis jirovecii pneumonia & $1(2)$ \\
\hline Wasting syndrome & $2(3)$ \\
\hline Tuberculosis & $1(2)$ \\
\hline \multicolumn{2}{|l|}{ Biomarkers } \\
\hline LPS plasma (pg/mL) & $102(78-121)$ \\
\hline sCD14 plasma (ng/mL) & $2382(1941-3111)$ \\
\hline sCD14 CSF (ng/mL) & $222(161-373)$ \\
\hline Neopterin plasma (nmol/L) & $12(9-23)$ \\
\hline Neopterin CSF (nmol/L) & $15(8-22)$ \\
\hline NFL CSF (ng/L) & $410(250-635)$ \\
\hline
\end{tabular}

Data is given as median (IQR), unless otherwise stated LPS lipopolysaccharide, NFL neurofilament light, CSF cerebrospinal fluid, MSM men who have sex with men, $I V$ intravenous

low CD4+ cell count were treated with trimetoprim/sulfametoxazole including four as prophylaxis against Pneumocystis jirovecii, and one patient was treated for Pneumocystis pneumonia. Furthermore, one patient was treated for tuberculosis.

The study was approved by the Regional Ethical Review Board in Gothenburg (Ö 588-01) and was performed in accordance with the Helsinki Declaration. All blood and CSF samples were obtained after informed consent of subjects under the IRB-approved protocol.

Markers of axonal damage, inflammation, and HIV-related factors

CSF NFL was measured using a sensitive sandwich enzyme-linked immunosorbent assay (ELISA) (NF-light ${ }^{\circ}$ ELISA kit, UmanDiagnostics AB, Umeå, Sweden) as previously described $[12,30]$. CSF neopterin was measured using a commercially available radioimmunoassay (Henningtest Neopterin, BRAHMS, Berlin, Germany) [15].
HIV-1 RNA in CSF and plasma were quantified with Roche Amplicor Monitor assay version 1.5 (Hoffman La-Roche, Basel, Switzerland) with a detection limit of 20 copies/mL. Furthermore, routine assessments included CSF white blood cell count and peripheral blood CD4+ cell count as previous described [31].

Valid measurement of neopterin in plasma and CSF, NFL and leucocyte count in CSF were available in 60, 61, 46, and 60 patients, respectively.

\section{Markers of microbial translocation and monocyte activation}

LPS in plasma and CSF was analysed by the Limulus amebocyte lysate colorimetric assay (Lonza, Walkersville, MD) according to the manufacturer's instructions and with the following modifications: Samples were diluted 10-fold to avoid interference with background colour, and preheated to $68{ }^{\circ} \mathrm{C}$ for $12 \mathrm{~min}$ prior to analysis to dissolve immune complexes, as previously described [32, 33].

Soluble CD14 in plasma and CSF was analysed using ELISA according to the manufacturer's instructions (R\&D, Minneapolis, MN).

\section{Statistics}

Data are given as median (interquartile range (IQR)). A $P$ value $\leq 0.05$ was considered significant. When comparing groups, the Student $T$-test and Mann Whitney $U$-test was used as appropriate. Uni- and multivariate linear regression models were used to assess associations and adjusted for possible confounders. When appropriate data were log-transformed (ln). In order to investigate possible associations between microbial translocation and inflammation in the CSF, multivariate linear regression models were performed with NFL and neopterin as the dependent variables, and sCD14 in the CSF was added as an independent variable. The model was adjusted for age, HIV RNA in the CSF, and CD4+ cell count. Since patients were included in two CD4+ cell count groups, CD4+ cell count was added to the model as both a continuous variable and as a nominal variable. Only the continuous variable was significant and therefore used in the final model. The analyses were performed using IBM SPSS 22.0 (Statistics for Windows, IBM Corp., Armonk, NY).

\section{Results}

\section{Characteristics of the study population}

The pilot study included 20 untreated, asymptomatic, HIV-infected patients with CSF pleocytosis as a sign of HIV-induced inflammation. Both LPS and sCD14 was detectable in all plasma samples. However, LPS was not detectable in any CSF samples whereas CSF-sCD14 was detectable in all CSF samples (data not shown). Hence, only sCD14 was measured in CSF in the subsequent analyses. 
Samples from 62 untreated, HIV-infected patients without clinical evidence of impaired cognitive function and with CD4+ cell count either above 350 or below 200 cells $/ \mu \mathrm{L}$ were selected for further analysis. Characteristics of the study population are shown in Table 1.

Detection of LPS in plasma and sCD14 in plasma and CSF Valid measurement of LPS in plasma and sCD14 in plasma was obtained in all 62 participants whereas sCD14 in CSF was obtained in 61 participants.

Patients with plasma sCD14 above median had higher plasma LPS compared to patients with sCD14 below median (91 pg/mL vs. $117 \mathrm{pg} / \mathrm{mL}, P=0.042)$, whereas there were no differences in age, gender, CD4+ cell count, or plasma HIV RNA between these two groups. However, when dividing the patients into two groups with SCD14 in CSF above or below median, no differences were found in regard to age, gender, CD4+ cell count, plasma HIV RNA, plasma LPS, or CFS HIV RNA (all $P$ values $>0.05$ ).

\section{Soluble CD14 and immune deficiency}

Increased concentrations of sCD14 in plasma was found in patients with CD4+ cell count $<200$ compared to patients with CD4+ cell count $>350$ (median of $2579 \mathrm{ng} / \mathrm{mL}$ (IQR
2061-3641) vs. 2357 ng/mL (IQR 1174-2959) $(P=0.031)$ ). Furthermore, concentration of sCD14 in CSF was 308 (IQR 196-385) in the group with CD4+ cell count $<200$ vs. 201 (IQR 90-317) in the group with CD4+ cell count $>350(P=0.015)$. Leucocyte cell counts in CSF were median 3 cells/ $\mu \mathrm{L}$ (IQR $1-11)$ and 5 cells/ $\mu \mathrm{L}$ (IQR 4-9) $(P=$ $0.406)$ in the group with CD $4+$ cell count $<200$ vs. the group with CD4+ cell count $>350$. Concentrations of CSF sCD14 was associated with CSF leucocyte cell count in a univariate analysis $(r=0.73, P=0.049)$.

\section{Markers of microbial translocation associated with inflammation in the CNS}

Univariate analyses demonstrated associations between plasma LPS and plasma sCD14 $(r=0.31, P=0.015)$, as well as borderline significant association with CSF sCD14 $(r=0.24, \quad P=0.059)$. Furthermore, plasma sCD14 was associated with CSF $\operatorname{sCD} 14(r=0.32, P=$ 0.012 , Fig. 1) and tended to correlate with plasma neopterin $(r=0.24, P=0.060)$. Importantly, CSF sCD14 was associated with a higher concentration of both NFL $(r=0.32, P=0.031)$ and neopterin in the CSF $(r=$ $0.32, P=0.012$, Fig. 1$)$.
A)

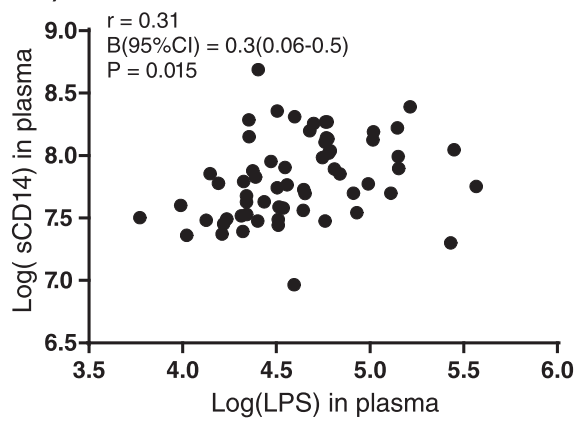

C)

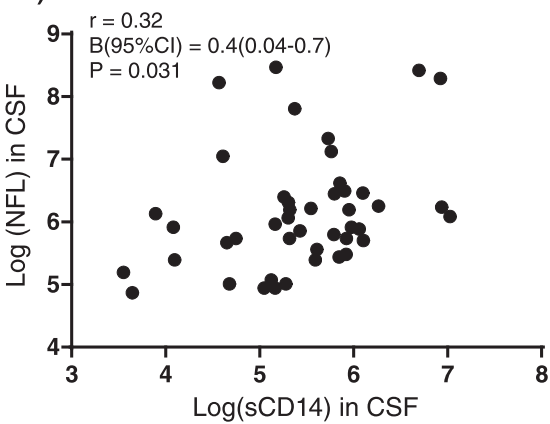

B)

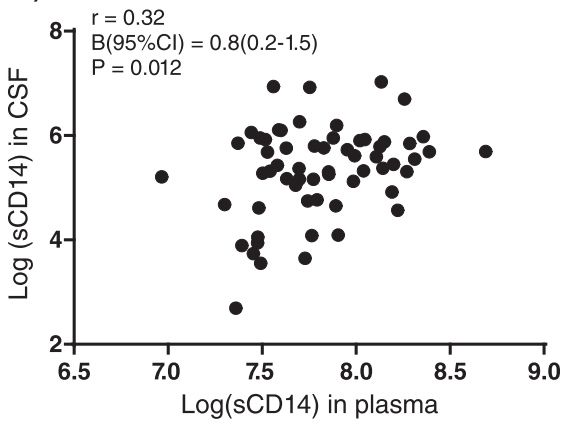

D)

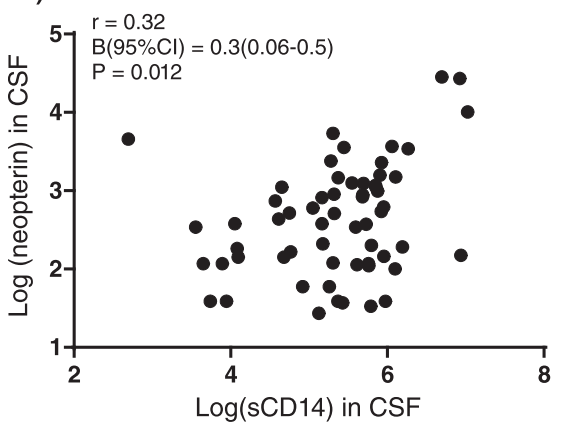

Fig. 1 Univariate analyses. Markers of microbial translocation (LPS), monocyte activation (sCD14), axonal damage (NFL), and CNS inflammation (neopterin) were measured in paired samples of cerebrospinal fluid (CSF) and plasma from 62 untreated HIV-infected patients. Univariate analysis were performed after Log(In) transformation due to skewed data. (a) plasma LPS and plasma sCD14, (b) plasma sCD14 and CSF sCD14, (c) CSF SCD14 and CSF NFL, (d) CSF SCD14 and CSF neopterin. CSF = cerebrospinal fluid, LPS = lipopolysaccharide, NFL = neurofilament light chain, sCD14 = soluble CD14 
In a multivariate linear regression model, CSF sCD14 remained associated with NFL $(P=0.039)$ and neopterin $(P=0.002)$ when adjusted for age, CD4+ cell count, and HIV RNA in CSF. In line with previous studies, higher age $(P=0.001)$ and lower $C D 4+$ cell count $(P=0.001)$ were associated with higher NFL in multivariable analysis [12]. Higher HIV RNA in CSF $(P<0.001)$ and lower CD4+ cell count $(P<0.001)$ were associated with higher neopterin. When LPS was added to the model, no association was found with NFL or neopterin.

\section{Discussion}

In this study of untreated, HIV-infected patients, sCD14, a marker of microbial translocation and monocyte activation was detectable in both plasma and CSF. High CSF and plasma sCD14 was not explained by higher HIV RNA or lower CD4+ cell counts, while a significant association between plasma sCD14 and LPS was found. Furthermore, significant associations between CSF sCD14 and markers of inflammation and axonal damage in the CSF were found, independent of age, HIV RNA, and CD4+ cell count. Hence, it is possible that elevated monocyte activation, partly driven by microbial translocation, may contribute to the pathogenesis in CNS by promoting inflammation.

Infection of the CNS occurs early during HIV infection $[34,35]$. HIV enters the CNS unassisted or in infected monocytes that cross the blood-brain barrier (BBB) [36-38]. In the CNS, HIV and migrated immune cells lead to the production of pro-inflammatory cytokines and further immune activation. Inflammation in the CNS creates a neurotoxic environment resulting in damage of the neurons [39]. Increased NFL reflects axonal damage [40], and neopterin is a marker of activated monocytes that is used to assess inflammation in the CNS in HIV-infected patients. In pre-cART studies high concentration of HIV RNA in the CSF was associated with severity of cognitive impairment [37, 38]. However, the association between HIV RNA and cognitive impairment in the cART era has not been as strong or consistent, indicating that other factors may also play a role in the development of HIV-associated cognitive impairment [38, 41-43].

Immune activation and inflammation is a hallmark of HIV infection and is caused by several factors including HIV replication, ART toxicity, co-infections, loss of regulatory cells, and microbial translocation [44]. Depletion of immune cells around the gastrointestinal tract leads to increased permeability for microbial products like LPS [45]. LPS is a part of the Gram negative bacterial wall and is frequently used in studies as a marker of microbial translocation. LPS cannot penetrate a normal functioning $\mathrm{BBB}$, however, inflammation caused by LPS may make the $\mathrm{BBB}$ more permeable for $\mathrm{HIV}$ and activated monocytes [46, 47]. To our knowledge LPS measurements in the CSF have not previously been reported.

The binding of LPS to TLR-4 results in secretion of pro-inflammatory cytokines and sCD14 from activated monocytes. We found significant associations between plasma LPS and plasma sCD14 as well as borderline significant association with CSF sCD14. Activated monocytes that have migrated to the CNS is the most likely source of sCD14 in the CSF, as SCD14 has not been reported to be produced from residing monocytes (microglia and astrocytes) [48]. Neopterin, which also is a marker of monocyte activation, is on the other hand produced by residing monocytes in the CSF [49]. This makes sCD14 an interesting biomarker in the CSF, as it potentially reflects the level of monocyte migration into the CNS supported by a positive correlation between CSF cell count and CSF sCD14. However, CD14 has been shown to possess several other functions besides binding LPS. CD14 also acts as a co-receptor for several other TLRs and microbial products which may explain the lack of consistency in studies investigating LPS in association with CD14 [50, 51].

Several studies have shown that markers of microbial translocation are associated with cognitive impairment in HIV-infected patients [7, 8, 52-54]. Thus, CNS HIV AntiRetroviral Therapy Effects Research (CHARTER) and The National NeuroAIDS Tissue Consortium (NNTC) groups have shown that LPS and sCD14 in plasma and in the CSF were increased in patients with impaired cognitive function $[7,8,53]$. Furthermore, increased sCD14 persists even after effective cART [55]. We found strong associations between CSF sCD14 and NFL, even after adjustment for age, HIV RNA, and CD4+ cell count, indicating that activated monocytes may contribute to axonal damage in the CNS, albeit this cross sectional study does not allow us to determine causality. Furthermore, our findings are supported by a recent study of CSF sCD14 and NFL in untreated HIV-infected patients [17].

Guidelines to assess cognitive function are based on the use of neuropsychological test [56]. Studies have shown that even in well treated HIV-infected individuals on CART, as many as $50 \%$ have been reported to have neurocognitive disorders [57-59]. However, neuropsychological tests are time consuming, require specialised staff, and are not available in all clinical settings. Furthermore, neuropsychological tests are not able to differentiate between active or residual cognitive impairment [60]. It has been suggested that a combination of biomarkers could be used to assess CNS inflammation and guide in diagnosing $\mathrm{HIV}$-associated CNS damage [61]. sCD14 could be a potential candidate as it has been established that activated monocytes play an important role in the pathogenesis of cognitive impairment in HIV 
infection [7, 8, 52-54], however in the present study markers of CNS inflammation and axonal damage was associated with CSF sCD14, but not with plasma sCD14. The present study did not include neurocognitive testing and only included naïve HIV-infected individuals. Data on neurocognitive performance as well as individuals on cART could add valuable information in future studies.

This study was limited by limited sample size and the cross-sectional design. Furthermore, measuring microbial translocation using LPS is challenging since the method is sensitive to both time and contamination [62]. However, this method is well established in our laboratory with an inter assay coefficient of variation $<10 \%$. Also, other microbial products besides LPS, including peptidoglycan, $\mathrm{Pam}_{3} \mathrm{CSK}_{4}$, polyI:C, and CpG DNA, cross the gastrointestinal mucosa and may activate the immune system and drive inflammation [50,51]. Recently it was found that two types of sCD14 exits [63]. A differential measurement of these may have provided additional information. Furthermore, neuropsychological evaluation would have supplied valuable information.

\section{Conclusion}

Significant associations between sCD14 in CSF and markers of CNS inflammation and axonal damage were found suggesting that monocyte activation, possibly driven by microbial translocation, may contribute to CNS inflammation. Further investigations into the pathogenesis of cognitive impairment in HIV infection, as well as potential biomarkers are needed, in order to increase the understanding and supply reliable methods of diagnosing these conditions.

\section{Ethics approval and consent to participate}

The study was approved by the Regional Ethical Review Board in Gothenburg (Ö 588-01) and was performed in accordance with the Helsinki Declaration. All blood and CSF samples were obtained after informed consent of subjects under the IRB-approved protocol.

\section{Consent for publication \\ Not applicable.}

\section{Availability of data and materials}

All data are available upon request.

\footnotetext{
Abbreviations

AIDS: acquired immunodeficiency syndrome; BBB: blood brain barrier; CART: combination antiretroviral treatment; CD: cluster of differentiation; CHARTER: CNS HIV Anti-Retroviral Therapy Effects Research; CNS: central nervous system; CSF: cerebrospinal fluid; ELISA: enzyme-linked immunosorbent assay; HIV: human immunodeficiency virus; IQR: interquartile range; IV: intravenous; LAL: limulus amebocyte lysate; LPS: lipopolysaccharide; mL: millilitre; mm3: cubic millimetre; $n$ : subsample size; NFL: neurofilament light chain protein; ng: nano gram; nmol: nano mole; NNTC: the National NeuroAIDS Tissue Consortium; Pg: Pico gram; RNA: ribonucleic acid; sCD14: soluble CD14; TLR: toll- like receptors.
}

\section{Competing interests}

The authors declare that they have no competing interests.

\section{Authors' contributions}

SJ participated in design of the study, performed statistical analysis, interpreted data, and drafted the manuscript. KKP participated in the design of the study, performed statistical analysis, and interpreted data. BA, MG and LH were responsible for the clinical data of the HIV-cohort. HZ performed the NFL ELISA. DF performed the neopterin immunoassay. MT participated in the design of the study, performed the SCD14 ELISA and LPS LAL colorimetric assay. SDN participated in the design of the study and interpreted data. All authors critically reviewed and approved the final manuscript.

\section{Acknowledgement}

We gratefully acknowledge patients that participated in this study.

\section{Funding}

SJ: Financial support from University of Copenhagen:

Internationaliseringspuljen.

KKP: Financial support from Rigshospitalets forskningspuljer, Augustinus Foundation, Snedkermester Sophus Jacobsen og hustru Astrid Jacobsens Foundation, Christian Larsen og dommer Ellen Larsens Legat, A.P. Møller, Fonden for lægevidenskabens Fremme, Aase and Ejnar Danielsens Foundation. BA: Financial support from the Sahlgrenska University Hospital (ALFGBG-430271) HZ: Financial support from European Research Council (681712), Swedish Research Council (2013-2546), Swedish State Support for Clinical Research (ALFGBG-441051) and the Knut and Alice Wallenberg Foundation. DF: No funding to be listed

MG: Financial support from the Sahlgrenska University Hospital (ALFGBG-430271) LH: Financial support from the Sahlgrenska University Hospital (ALFGBG-430271) MT: Financial support from Rigshospitalet and Oslo University Hospital SDN: Financial support from Rigshospitalet, Funding Novo Nordisk Foundation, Åse og Ejnar Danielsens Fondation and Augustinus Foundation.

\section{Author details}

'Department of Infectious Diseases, Viro-immunology Research Unit, Rigshospitalet, University of Copenhagen, Blegdamsvej 9, DK 2100 Copenhagen $\varnothing$, Denmark. ${ }^{2}$ Department of Infectious Diseases, Institute of Biomedicine, Sahlgrenska Academy, University of Gothenburg, Gothenburg, Sweden. ${ }^{3}$ Clinical Neurochemistry Laboratory, Institute of Neuroscience and Physiology, Sahlgrenska Academy, University of Gothenburg, Mölndal, Sweden. ${ }^{4}$ Department of Molecular Neuroscience, UCL Institute of Neurology, Queen Square, London, UK. ${ }^{5}$ Division of Biological Chemistry, Biocenter, Innsbruck Medical University, Innsbruck, Austria. ${ }^{6}$ Department of Infectious Diseases, Oslo University Hospital, Oslo, Norway.

Received: 29 December 2015 Accepted: 13 April 2016

Published online: 21 April 2016

\section{References}

1. Grant I, Atkinson JH, Hesselink JR, et al. Evidence for early central nervous system involvement in the acquired immunodeficiency syndrome (AIDS) and other human immunodeficiency virus (HIV) infections. Studies with neuropsychologic testing and magnetic resonance imaging. Ann Intern Med. 1987;107:828-36.

2. Levy RM, Janssen RS, Bush TJ, Rosenblum ML. Neuroepidemiology of acquired immunodeficiency syndrome. J Acquir Immune Defic Syndr. 1988;1:31-40.

3. McArthur JC. HIV dementia: an evolving disease. J Neuroimmunol. 2004;157:3-10.

4. Maschke M, Kastrup O, Esser S, Ross B, Hengge U, Hufnagel A. Incidence and prevalence of neurological disorders associated with HIV since the introduction of highly active antiretroviral therapy (HAART). J Neurol Neurosurg Psychiatry. 2000;69:376-80.

5. Sevigny JJ, Albert SM, McDermott MP, et al. Evaluation of HIV RNA and markers of immune activation as predictors of HIV-associated dementia. Neurology. 2004;63:2084-90.

6. Ragin $A B, W u Y, O c h s ~ R$, et al. Biomarkers of neurological status in HIV infection: a 3-year study. Proteomics Clin Appl. 2010;4:295-303.

7. Lyons JL, Uno H, Ancuta P, et al. Plasma SCD14 is a biomarker associated with impaired neurocognitive test performance in attention and learning domains in HIV infection. J Acquir Immune Defic Syndr. 2011;57:371-9. 
8. Ancuta P, Kamat A, Kunstman K, et al. Microbial translocation is associated with increased monocyte activation and dementia in AIDS patients. PLoS One. 2008;3, e2516.

9. Hagberg L, Cinque P, Gisslen M, et al. Cerebrospinal fluid neopterin: an informative biomarker of central nervous system immune activation in HIV1 infection. AIDS Res Ther. 2010;7:15.

10. Burdo TH, Weiffenbach A, Woods SP, Letendre S, Ellis RJ, Williams KC. Elevated SCD163 in plasma but not cerebrospinal fluid is a marker of neurocognitive impairment in HIV infection. AIDS. 2013;27:1387-95.

11. Yilmaz A, Yiannoutsos CT, Fuchs D, et al. Cerebrospinal fluid neopterin decay characteristics after initiation of antiretroviral therapy. J Neuroinflammation. 2013;10:62.

12. Jessen KJ, Mellberg T, Price RW, et al. Biomarker evidence of axonal injury in neuroasymptomatic HIV-1 patients. PLoS One. 2014;9, e88591.

13. Peluso MJ, Meyerhoff DJ, Price RW, et al. Cerebrospinal fluid and neuroimaging biomarker abnormalities suggest early neurological injury in a subset of individuals during primary HIV infection. J Infect Dis. 2013;207:1703-12.

14. Gisslen M, Hagberg L, Brew BJ, Cinque P, Price RW, Rosengren L. Elevated cerebrospinal fluid neurofilament light protein concentrations predict the development of AIDS dementia complex. J Infect Dis. 2007;195:1774-8.

15. Mellgren A, Price RW, Hagberg L, Rosengren L, Brew BJ, Gisslen M. Antiretroviral treatment reduces increased CSF neurofilament protein (NFL) in HIV-1 infection. Neurology. 2007;69:1536-41.

16. Gisslen M, Rosengren L, Hagberg L, Deeks SG, Price RW. Cerebrospinal fluid signs of neuronal damage after antiretroviral treatment interruption in HIV-1 infection. AIDS Res Ther. 2005;2:6.

17. McGuire JL, Gill AJ, Douglas SD, Kolson DL. Central and peripheral markers of neurodegeneration and monocyte activation in HIV-associated neurocognitive disorders. J Neurovirol. 2015;21:439-48.

18. Neuhaus J, Jacobs Jr DR, Baker JV, et al. Markers of inflammation, coagulation, and renal function are elevated in adults with HIV infection. J Infect Dis. 2010;201:1788-95.

19. Armah KA, McGinnis K, Baker J, et al. HIV status, burden of comorbid disease, and biomarkers of inflammation, altered coagulation, and monocyte activation. Clin Infect Dis. 2012;55:126-36.

20. Pedersen KK, Pedersen M, Gaardbo JC, et al. Persisting inflammation and chronic immune activation but intact cognitive function in HIV-infected patients after long-term treatment with combination antiretroviral therapy. J Acquir Immune Defic Syndr. 2013;63:272-9.

21. Marchetti G, Cozzi-Lepri A, Merlini E, et al. Microbial translocation predicts disease progression of HIV-infected antiretroviral-naive patients with high CD4+ cell count. AIDS. 2011;25:1385-94.

22. Dandekar S. Pathogenesis of HIV in the gastrointestinal tract. Curr HIV/AIDS Rep. 2007:4:10-5.

23. Sandler NG, Wand H, Roque A, et al. Plasma levels of soluble CD14 independently predict mortality in HIV infection. J Infect Dis. 2011;203:780-90.

24. Epple HJ, Schneider T, Troeger H, et al. Impairment of the intestinal barrier is evident in untreated but absent in suppressively treated HIV-infected patients. Gut. 2009:58:220-7.

25. Brenchley JM, Paiardini M, Knox KS, et al. Differential Th17 CD4 T-cell depletion in pathogenic and nonpathogenic lentiviral infections. Blood. 2008;112:2826-35.

26. Guadalupe M, Reay E, Sankaran S, et al. Severe CD4+ T-cell depletion in gut lymphoid tissue during primary human immunodeficiency virus type 1 infection and substantial delay in restoration following highly active antiretroviral therapy. J Virol. 2003;77:11708-17.

27. Mehandru S, Poles MA, Tenner-Racz K, et al. Primary HIV-1 infection is associated with preferential depletion of CD4+ T lymphocytes from effector sites in the gastrointestinal tract. J Exp Med. 2004;200:761-70.

28. Brenchley JM, Schacker TW, Ruff LE, et al. CD4+ T cell depletion during all stages of HIV disease occurs predominantly in the gastrointestinal tract. J Exp Med. 2004;200:749-59.

29. Dauphinee SM, Karsan A. Lipopolysaccharide signaling in endothelial cells. Lab Invest. 2006;86:9-22.

30. Rosengren LE, Karlsson JE, Karlsson JO, Persson LI, Wikkelso C. Patients with amyotrophic lateral sclerosis and other neurodegenerative diseases have increased levels of neurofilament protein in CSF. J Neurochem. 1996;67: 2013-8.

31. Hagberg L, Dotevall L, Norkrans G, Larsson M, Wachter H, Fuchs D. Cerebrospinal fluid neopterin concentrations in central nervous system infection. J Infect Dis. 1993;168:1285-8.
32. Troseid M, Nowak P, Nystrom J, Lindkvist A, Abdurahman S, Sonnerborg A. Elevated plasma levels of lipopolysaccharide and high mobility group box-1 protein are associated with high viral load in HIV-1 infection: reduction by 2-year antiretroviral therapy. AIDS. 2010;24:1733-7.

33. Manner IW, Baekken M, Kvale D, et al. Markers of microbial translocation predict hypertension in HIV-infected individuals. HIV Med. 2013;14:354-61.

34. Davis LE, Hjelle BL, Miller VE, et al. Early viral brain invasion in iatrogenic human immunodeficiency virus infection. Neurology. 1992;42:1736-9.

35. Valcour V, Chalermchai T, Sailasuta N, et al. Central nervous system viral invasion and inflammation during acute HIV infection. J Infect Dis. 2012;206:275-82.

36. Banks WA, Freed EO, Wolf KM, Robinson SM, Franko M, Kumar VB. Transport of human immunodeficiency virus type 1 pseudoviruses across the bloodbrain barrier: role of envelope proteins and adsorptive endocytosis. J Virol. 2001;75:4681-91.

37. Brew BJ, Pemberton L, Cunningham P, Law MG. Levels of human immunodeficiency virus type 1 RNA in cerebrospinal fluid correlate with AIDS dementia stage. J Infect Dis. 1997;175:963-6.

38. Ellis RJ, Moore DJ, Childers ME, et al. Progression to neuropsychological impairment in human immunodeficiency virus infection predicted by elevated cerebrospinal fluid levels of human immunodeficiency virus RNA. Arch Neurol. 2002;59:923-8.

39. Kovalevich J, Langford D. Neuronal toxicity in HIV CNS disease. Future Virol. 2012;7:687-98.

40. Jonsson M, Zetterberg $\mathrm{H}$, van SE, et al. Cerebrospinal fluid biomarkers of white matter lesions - cross-sectional results from the LADIS study. Eur J Neurol. 2010;17:377-82.

41. Nightingale S, Winston A, Letendre S, et al. Controversies in HIV-associated neurocognitive disorders. Lancet Neurol. 2014;13:1139-51.

42. Valcour VG. Evaluating cognitive impairment in the clinical setting: practical screening and assessment tools. Top Antivir Med. 2011;19:175-80.

43. Tyler KL, MCArthur JC. Through a glass, darkly: cerebrospinal fluid viral load measurements and the pathogenesis of human immunodeficiency virus infection of the central nervous system. Arch Neurol. 2002;59:909-12.

44. Deeks SG, Lewin SR, Havlir DV. The end of AIDS: HIV infection as a chronic disease. Lancet. 2013;382:1525-33.

45. Brenchley JM, Price DA, Schacker TW, et al. Microbial translocation is a cause of systemic immune activation in chronic HIV infection. Nat Med. 2006;12: 1365-71.

46. Zhou H, Andonegui G, Wong CH, Kubes P. Role of endothelial TLR4 for neutrophil recruitment into central nervous system microvessels in systemic inflammation. J Immunol. 2009;183:5244-50.

47. Banks WA, Gray AM, Erickson MA, et al. Lipopolysaccharide-induced bloodbrain barrier disruption: roles of cyclooxygenase, oxidative stress, neuroinflammation, and elements of the neurovascular unit. J Neuroinflammation. 2015;12:223.

48. Cauwels A, Frei K, Sansano S, et al. The origin and function of soluble CD14 in experimental bacterial meningitis. J Immunol. 1999;162:4762-72.

49. Brew BJ, Bhalla RB, Paul M, et al. Cerebrospinal fluid neopterin in human immunodeficiency virus type 1 infection. Ann Neurol. 1990;28:556-60.

50. Lien E, Aukrust P, Sundan A, Muller F, Froland SS, Espevik T. Elevated levels of serum-soluble CD14 in human immunodeficiency virus type 1 (HIV-1) infection: correlation to disease progression and clinical events. Blood. 1998;92:2084-92.

51. Lee CC, Avalos AM, Ploegh HL. Accessory molecules for Toll-like receptors and their function. Nat Rev Immunol. 2012;12:168-79.

52. Ryan LA, Zheng J, Brester M, et al. Plasma levels of soluble CD14 and tumor necrosis factor-alpha type II receptor correlate with cognitive dysfunction during human immunodeficiency virus type 1 infection. J Infect Dis. 2001;184:699-706.

53. Kamat A, Lyons JL, Misra V, et al. Monocyte activation markers in cerebrospinal fluid associated with impaired neurocognitive testing in advanced HIV infection. J Acquir Immune Defic Syndr. 2012;60:234-43.

54. Fischer-Smith T, Croul S, Sverstiuk AE, et al. CNS invasion by CD14+/CD16+ peripheral blood-derived monocytes in HIV dementia: perivascular accumulation and reservoir of HIV infection. J Neurovirol. 2001;7:528-41.

55. Hattab S, Guiguet M, Carcelain G, et al. Soluble biomarkers of immune activation and inflammation in HIV infection: impact of 2 years of effective first-line combination antiretroviral therapy. HIV Med. 2015.

56. Antinori A, Arendt G, Becker JT, et al. Updated research nosology for HIVassociated neurocognitive disorders. Neurology. 2007;69:1789-99.

57. Robertson KR, Smurzynski M, Parsons TD, et al. The prevalence and incidence of neurocognitive impairment in the HAART era. AIDS. 2007;21: 1915-21. 
58. Lescure FX, Omland LH, Engsig FN, et al. Incidence and impact on mortality of severe neurocognitive disorders in persons with and without HIV infection: a Danish nationwide cohort study. Clin Infect Dis. 2011;52:235-43.

59. Heaton RK, Franklin Jr DR, Deutsch R, et al. Neurocognitive change in the Era of HIV combination antiretroviral therapy: the longitudinal CHARTER study. Clin Infect Dis. 2015:60:473-80.

60. Price RW, Peterson J, Fuchs D, et al. Approach to cerebrospinal fluid (CSF) biomarker discovery and evaluation in HIV infection. J Neuroimmune Pharmacol. 2013;8:1147-58.

61. Gisslen M, Hagberg L, Rosengren L, et al. Defining and evaluating HIVrelated neurodegenerative disease and its treatment targets: a combinatorial approach to use of cerebrospinal fluid molecular biomarkers. J Neuroimmune Pharmacol. 2007;2:112-9.

62. Marchetti G, Tincati C, Silvestri G. Microbial translocation in the pathogenesis of HIV infection and AIDS. Clin Microbiol Rev. 2013:26:2-18.

63. Lodrup Carlsen KC, Granum B. Soluble CD14: role in atopic disease and recurrent infections, including otitis media. Curr Allergy Asthma Rep. 2007;7:436-43.

Submit your next manuscript to BioMed Central and we will help you at every step:

- We accept pre-submission inquiries

- Our selector tool helps you to find the most relevant journal

- We provide round the clock customer support

- Convenient online submission

- Thorough peer review

- Inclusion in PubMed and all major indexing services

- Maximum visibility for your research

Submit your manuscript at www.biomedcentral.com/submit
Biomed Central 